PROCEEDINGS OF THE

AMERICAN MATHEMATICAL SOCIETY

Volume 131, Number 3, Pages 755-759

S 0002-9939(02)06864-8

Article electronically published on October 18, 2002

\title{
ON THE BIEBERBACH CONJECTURE AND HOLOMORPHIC DYNAMICS
}

\author{
XAVIER BUFF
}

(Communicated by Linda Keen)

\begin{abstract}
In this note we prove that when $P$ is a polynomial of degree $d$ with connected Julia set and when $z_{0}$ belongs to the filled-in Julia set $K(P)$, then $\left|P^{\prime}\left(z_{0}\right)\right| \leq d^{2}$. We also show that equality is achieved if and only if $K(P)$ is a segment of which one extremity is $z_{0}$. In that case, $P$ is conjugate to a Tchebycheff polynomial or its opposite. The main tool in our proof is the Bieberbach conjecture proved by de Branges in 1984 .
\end{abstract}

\section{INTRODUCTION}

Let us first recall two well-known dynamical results which are in the same vein as ours.

Theorem 1. Let $P$ be a monic centered polynomial with connected Julia set. Then, for any $z_{0} \in K(P)$, we have $\left|z_{0}\right| \leq 2$ with equality if and only if $K(P)$ is a segment of which one extremity is $z_{0}$.

Proof. Assume $K$ is a compact connected subset of $\mathbb{C}$ and $\mathbb{C} \backslash K$ is conformally isomorphic to $\mathbb{C} \backslash \overline{\mathbb{D}}$. Let $\phi: \mathbb{C} \backslash \overline{\mathbb{D}} \rightarrow \mathbb{C} \backslash K$ be a conformal isomorphism with Laurent series expansion

$$
\phi(z)=b_{1} z+b_{0}+\frac{b_{-1}}{z}+\frac{b_{-2}}{z^{2}}+\ldots .
$$

Then, the Gronwall Area Formula asserts that the area of $K$ is equal to $\pi \sum_{n \leq 1} n\left|b_{n}\right|^{2}$. It follows that $\left|b_{1}\right| \leq\left|b_{-1}\right|$, with equality if and only if $K$ is a straight line segment. Moreover, when $b_{0}=0$ and $z_{0} \in K$, by considering the map $\psi(w)=\sqrt{\phi\left(w^{2}\right)-z_{0}}$, we get $\left|z_{0}\right| \leq 2\left|b_{1}\right|$ with equality if and only if $K$ is a straight line segment of which one extremity is $z_{0}$.

Then, observe that when $P$ is a monic centered polynomial, the Böttcher coordinate $\phi: \mathbb{C} \backslash \overline{\mathbb{D}} \rightarrow \mathbb{C} \backslash K(P)$ has Laurent series expansion of the form

$$
\phi(z)=z+\frac{b_{-1}}{z}+\frac{b_{-2}}{z^{2}}+\ldots
$$

Indeed, $b_{1}=1$ because $P$ is monic and $b_{0}=0$ because $P$ is centered. Theorem 1 follows immediately.

Received by the editors June 25, 2001 and, in revised form, August 14, 2001.

2000 Mathematics Subject Classification. Primary 37F10, 30C50.

(C)2002 American Mathematical Society 
Theorem 2. Let $P$ be a polynomial of degree $d$ with connected Julia set. If $\alpha$ is a fixed point of $P$, then $\left|P^{\prime}(\alpha)\right| \leq d^{2}$.

This is a weak version of an inequality due to Pommerenke $[\mathrm{Po}$, Levine $\mathrm{L}]$ and Yoccoz $[\mathrm{Y}]$ (see $[\mathrm{H}]$ or $[\mathrm{Pe}]$ ). The idea of the proof goes back to Bers's Inequality in the context of quasi-fuchsian groups. There, Bers proves that the length of a hyperbolic geodesic in $Q(X, Y)$ is bounded by the hyperbolic length of the corresponding geodesic on $X$ or $Y$ (see [B] Theorem 3 and [McM] Prop. 6.4). In [O] Sect. 5.1, Otal gives a proof of Bers's Inequality based on Koebe's One-Quarter Theorem. His proof is inspired by Ahlfors (see A Lemma 1).

In the present article, we present a generalization of those two theorems. We will use the Bieberbach conjecture proved by de Branges in 1984 .

De Branges's Theorem. Let $\phi: \mathbb{D} \rightarrow \mathbb{C}$ be a univalent mapping. If $\phi(z)=$ $\sum_{n \geq 1} a_{n} z^{n}$, then for any $n \geq 1$, we have $\left|a_{n} / a_{1}\right| \leq n$. Besides, if $\left|a_{k} / a_{1}\right|=k$ for some integer $k>1$, then $\phi$ is a rotation of the Koebe function, i.e., there exists a real $\theta$ such that

$$
\phi(z)=\frac{z}{\left(1-e^{i \theta} z\right)^{2}} .
$$

We obtain a result which does not only control the derivative of $P$ at its fixed points, but controls the derivative of $P$ at all the points in the Julia set. Our main observation is the following.

Lemma 1. Let $f:(\mathbb{C}, 0) \rightarrow(\mathbb{C}, 0)$ be a germ such that 0 is a superattracting fixed point with local degree $k \geq 2$. Let $\phi:(\mathbb{C}, 0) \rightarrow(\mathbb{C}, 0)$ be a Böttcher coordinate, i.e., a germ which is univalent in a neighborhood of 0 and which satisfies $\phi\left(z^{k}\right)=f(\phi(z))$ for $z$ sufficiently close to 0 . If $\phi(z)=\sum_{n \geq 1} a_{n} z^{n}$, then

$$
\operatorname{res}\left(\frac{1}{f}, 0\right)=k \frac{a_{k}}{a_{1}} \text {. }
$$

Remark. The result still holds if instead of germs one considers formal power series, but we are not aware of a formal proof.

We say that a polynomial $P$ is a Tchebycheff polynomial if $P(\cos z)=\cos (d z)$, where $d$ is the degree of $P$. As a corollary of Lemma 1] we will show the following two theorems.

Theorem 3. Assume $P$ is a polynomial of degree $d$ with connected Julia set. Then, for any $z_{0} \in K(P)$, we have $\left|P^{\prime}\left(z_{0}\right)\right| \leq d^{2}$ with equality if and only if $K(P)$ is a segment, one extremity of which is $z_{0}$. In that case, $P$ is conjugate to a Tchebycheff polynomial or to its opposite.

Theorem 4. Assume $P$ is a polynomial of degree $d$ with disconnected Julia set. Let $g_{P}: \mathbb{C} \rightarrow \mathbb{R}^{+}$be the Green's function of $K(P)$ and set

$$
G(P)=\max _{\left\{\omega \mid P^{\prime}(\omega)=0\right\}} g_{P}(\omega) .
$$

Then, for any $z_{0} \in \mathbb{C}$ with $g_{P}\left(z_{0}\right) \leq G(P)$, we have $\left|P^{\prime}\left(z_{0}\right)\right|<d^{2} e^{(d-1) G(P)}$.

Remark. This inequality always holds for points in $K(P)$. 


\section{Proofs of the Results}

Proof of Lemma 1. Let $\gamma_{1}$ be a small circle around 0 and let $\gamma_{2}$ be its image by $\phi$. Then,

$$
\operatorname{res}\left(\frac{1}{f}, 0\right)=\int_{\gamma_{2}} \frac{d w}{f(w)} \underset{w=\phi(z)}{=} \int_{\gamma_{1}} \frac{\phi^{\prime}(z)}{f(\phi(z))} d z=\int_{\gamma_{1}} \frac{\phi^{\prime}(z)}{\phi\left(z^{k}\right)} d z=\operatorname{res}\left(\frac{\phi^{\prime}(z)}{\phi\left(z^{k}\right)}, 0\right) .
$$

Since $\phi(z)=\sum_{n \geq 1} a_{n} z^{n}$, we have

$$
\frac{\phi^{\prime}(z)}{\phi\left(z^{k}\right)}=\frac{a_{1}+2 a_{2} z+\ldots+k a_{k} z^{k-1}+\mathcal{O}\left(|z|^{k}\right)}{a_{1} z^{k}\left(1+\mathcal{O}\left(|z|^{k}\right)\right)}=\frac{1}{z^{k}}+\frac{2 a_{2}}{a_{1}} \frac{1}{z^{k-1}}+\ldots+\frac{k a_{k}}{a_{1}} \frac{1}{z}+\mathcal{O}(1) .
$$

Therefore

$$
\operatorname{res}\left(\frac{1}{f}, 0\right)=\operatorname{res}\left(\frac{\phi^{\prime}(z)}{\phi\left(z^{k}\right)}, 0\right)=\frac{k a_{k}}{a_{1}} .
$$

Proof of Theorem 0 . First, observe that when $P$ is conjugate to a Tchebycheff polynomial of degree $d$ (or its opposite), $K(P)$ is a segment and the derivative at an extremity is $\pm d^{2}$. The proof is not difficult and left to the reader.

Next, assume $P$ is a polynomial of degree $d$ with connected Julia set and $z_{0}$ belongs to the filled-in Julia set $K(P)$. Let $\Omega$ be the simply connected sub-domain of $\mathbb{P}^{1}$ defined by

$$
\Omega=\left\{w \in \mathbb{P}^{1} \mid z_{0}+\frac{1}{w} \in \mathbb{P}^{1} \backslash K(P)\right\} .
$$

Since $z_{0} \in K(P)$, we see that $\Omega \subset \mathbb{C}$, and since $P$ has a superattracting fixed point with local degree $d$ at infinity, the rational map $f: \mathbb{P}^{1} \rightarrow \mathbb{P}^{1}$ defined by

$$
f(w)=\frac{1}{P\left(z_{0}+1 / w\right)-z_{0}}
$$

has a superattracting fixed point at 0 with local degree $d$. Any Böttcher coordinate of $f$ extends to a univalent mapping $\phi: \mathbb{D} \rightarrow \Omega$ and Lemma 1 asserts that writing $\phi(z)=\sum_{n \geq 1} a_{n} z^{n}$, we get

$$
d \frac{a_{d}}{a_{1}}=\operatorname{res}\left(\frac{1}{f}, 0\right) .
$$

Since $P(z)=b_{0}+b_{1}\left(z-z_{0}\right)+\ldots+b_{d}\left(z-z_{0}\right)^{d}$, we see that

$$
\frac{1}{f(w)}=P\left(z_{0}+1 / w\right)-z_{0}=b_{0}-z_{0}+\frac{b_{1}}{w}+\ldots+\frac{b_{d}}{w^{d}} .
$$

Therefore, res $(1 / f, 0)=b_{1}=P^{\prime}\left(z_{0}\right)$. It now follows from de Branges's Theorem that

$$
\left|P^{\prime}\left(z_{0}\right)\right|=\left|d \frac{a_{d}}{a_{1}}\right| \leq d^{2},
$$

with equality if and only if $\phi$ is a rotation of the Koebe function. In that case, $\Omega$ is a slit plane, and thus $K(P)$ is a segment of which one extremity is $z_{0}$.

We must now show that $P$ is conjugate to a Tchebycheff polynomial or to its opposite. Knowing that $K(P)$ is a segment, this is classical. Conjugating $P$ with an affine map, we may assume that $K(P)=[-1,1]$. We define $\psi: \mathbb{P}^{1} \backslash \overline{\mathbb{D}} \rightarrow \mathbb{P}^{1} \backslash[-1,1]$ to be the conformal representation

$$
\psi(z)=\frac{1}{2}\left(z+\frac{1}{z}\right) .
$$


The conformal representation $\psi^{-1}: \mathbb{P}^{1} \backslash[-1,1] \rightarrow \mathbb{P}^{1} \backslash \overline{\mathbb{D}}$ conjugates the proper mapping $P: \mathbb{P}^{1} \backslash[-1,1] \rightarrow \mathbb{P}^{1} \backslash[-1,1]$ to a proper mapping from $\mathbb{P}^{1} \backslash \overline{\mathbb{D}}$ to itself, having a superattracting fixed point of degree $d$ at infinity. This mapping is necessarily of the form $z \mapsto \lambda z^{d}$, with $|\lambda|=1$.

Since $K(P)$ is totally invariant, the polynomial $P$ necessarily maps the set $\{-1,1\}$ into itself. Besides, $\psi^{-1}(z)$ tends to \pm 1 as $z$ tends to \pm 1 . Therefore, the map $z \mapsto \lambda z^{d}$ maps the set $\{-1,1\}$ into itself. This shows that $\lambda= \pm 1$. Hence,

$$
\left(\forall z \in \mathbb{P}^{1} \backslash \overline{\mathbb{D}}\right) \quad P(\psi(z))=\psi\left( \pm z^{d}\right) .
$$

As $z \rightarrow e^{i \theta} \in S^{1}$, we get

$$
P(\cos \theta)=P\left(\frac{e^{i \theta}+e^{-i \theta}}{2}\right)= \pm \frac{e^{i d \theta}+e^{-i d \theta}}{2}= \pm \cos (d \theta) .
$$

Proof of Theorem 4 . We will mimick the previous proof. We assume that $g_{P}\left(z_{0}\right) \leq$ $G(P)$ and we set

$$
\Omega=\left\{w \in \mathbb{P}^{1} \mid g_{P}\left(z_{0}+\frac{1}{w}\right)>G(P)\right\} .
$$

We define $f: \mathbb{P}^{1} \rightarrow \mathbb{P}^{1}$ by $f(w)=1 /\left(P\left(z_{0}+1 / w\right)-z_{0}\right)$. Then the Böttcher coordinate of $f$ at 0 extends to a univalent mapping $\phi$ between the disk centered at 0 with radius $e^{-G(P)}$ and the domain $\Omega \subset \mathbb{C}$. Since the mapping

$$
z \mapsto \phi\left(e^{-G(P)} z\right)=\sum_{n \geq 1} a_{n} e^{-n G(P)} z^{n}
$$

is univalent in the unit disk, de Branges's Theorem only allows us to conclude that

$$
\left|P^{\prime}\left(z_{0}\right)\right|=d\left|\frac{a_{d}}{a_{1}}\right|=d e^{(d-1) G(P)}\left|\frac{a_{d} e^{-d G(P)}}{a_{1} e^{-G(P)}}\right|<d^{2} e^{(d-1) G(P)} .
$$

The inequality is strict because the complement of $\Omega$ has non-empty interior, and therefore, $\Omega$ cannot be a slit plane.

\section{APPLiCATION}

A possible application of Theorem 3 is the following.

Corollary 1. Let $d \geq 3$ be an integer, and $a=\left(a_{2}, \ldots, a_{d-1}\right)$ be a point in $\mathbb{C}^{d-2}$. Then, the Julia set of the polynomial $P_{a}(z)=d^{2} z+a_{2} z^{2}+\ldots+a_{d-1} z^{d-1}+z^{d}$ is connected if and only if $P_{a}$ is conjugate to a Tchebycheff polynomial.

Proof. On the one hand, if $P_{a}$ is conjugate to a Tchebycheff polynomial, its Julia set is a segment and therefore it is connected. On the other hand, observe that 0 is a fixed point with multiplier $d^{2}$. Therefore, Theorem 3 shows that if $J\left(P_{a}\right)$ is connected, then $P_{a}$ is conjugate to a Tchebycheff polynomial or its opposite and 0 is an extremity of $K\left(P_{a}\right)$. Since 0 is fixed, $P_{a}$ may always be conjugate to a Tchebycheff polynomial.

Every polynomial of degree $d$ having a fixed point with multiplier $d^{2}$ is conjugate to a polynomial $P_{a}$. The family $\left(P_{a}\right)_{a \in \mathbb{C}^{d-2}}$ is a co-dimension 1 algebraic sub-variety of the space of polynomials up to conjugacy. The set of polynomials $P_{a}$ which are conjugate to a Tchebycheff polynomial is finite but not empty. Therefore, for 
each degree $d \geq 3$, we produce an example of co-dimension 1 algebraic family of polynomials for which the connectivity locus is non-empty and discrete.

\section{ACKNOWLEDGEMENTS}

The author wishes to express his gratitude to Gilbert Levitt and Jean-Pierre Ramis for fruitful discussions.

\section{REFERENCES}

[A] L. Ahlfors. Finitely generated Kleinian groups. Amer. J. Math. 86 (1964) 413-429. MR 29:4890

[B] L. Bers. On Boundaries of Teichmüller Spaces and on Kleinian Groups. Annals of Math. 91 (1970) 570-600. MR 45:7044

$[\mathrm{H}] \quad$ J.H. HubBARD, Local connectivity of Julia sets and bifurcation loci: three theorems of J.C. Yoccoz, in Topological Methods in Modern Mathematics, L.R. Goldberg and A.V. Phillips eds, Publish or Perish, 467-511 (1993). MR 94c:58172

[L] G. M. Levin. On Pommerenke's inequality for the eigenvalues of fixed points. Coll. Mathematicum LXII (1991), 167-177. MR 93h:30053

[McM] C.T. McMullen, Iteration on Teichmüller space, Inv. Math. 99 (1989), 425-454. MR 91a:57008

[M] J. Milnor. Dynamics in one complex variable, Introductory Lectures. Friedr. Vieweg \& Sohn, Braunschweig (1999). MR 2001i:37057

[O] J.P. OtaL. Le théorème d'hyperbolisation pour les variétés fibrées de dimension 3. Astérisque 235 (1996). MR 97e:57013

[Pe] C.L. Petersen, On the Pommerenke, Levin Yoccoz inequality, Ergod. Th. \& Dynan. Sys. 13 (1993), 785-806. MR 94k:58122

[Po] Ch. Pommerenke. On conformal mapping and iteration of rational functions. Complex Variables Th. and Appl. 5 (1986), 117-126. MR 87i:30045

[Y] J.C. Yoccoz. Sur la taille des membres de l'Ensemble de Mandelbrot. (1987) (unpublished).

Laboratoire Emile Picard, Université Paul Sabatier, 118, route de Narbonne, 31062 Toulouse Cedex, France

E-mail address: buff@picard.ups-tlse.fr 